\title{
Supporting Connectivism in Knowledge Based Engineering with Graph Theory, Filtering Techniques and Model Quality Assurance
}

\author{
Joel Johansson, Manuel Contero, Pedro Company, Fredrik Elgh
}

\begin{abstract}
Mass-customization has forced manufacturing companies to put significant efforts to digitize and automate their engineering and production processes. When new products are to be developed and introduced the production is not alone to be automated. The application of knowledge regarding how the product should be designed and produced based on customer requirements also must be automated. One big academic challenge is helping industry to make sure that the background knowledge of the automated engineering processes still can be understood by its stakeholders throughout the product life cycle.

The research presented in this paper aims to build an infrastructure to support a connectivistic view on knowledge in knowledge based engineering. Fundamental concepts in connectivism include network formation and contextualization, which are here addressed by using graph theory together with information filtering techniques and quality assurance of CAD-models. The paper shows how engineering knowledge contained in spreadsheets, knowledge-bases and CAD-models can be penetrated and represented as filtered graphs to support a connectivistic working approach. Three software demonstrators developed to extract filtered graphs are presented and discussed in the paper.
\end{abstract}

Keywords: Connectivism, mass-customization, knowledge management, knowledge-based engineering, graph theory

\section{Introduction}

Engineering knowledge refers to the knowledge that engineers apply when they are involved in developing products and their corresponding production systems. This broad definition excludes curiosities and emphasizes applicability, as long as it implies that the knowledge is part of decision-making processes. Engineering knowledge further refers to any reason for why, how, when, where, what, and by whom something is to be done or constituted. The sum of engineering knowledge formally represented for one product is referred to as product knowledge and may reside in any available product representation, provided that it is made available and interrelated.

The practical need to capture and automatically utilize such a wide and disperse amount of engineering knowledge derives from the fact that describing the purpose of a design and the justifications for specific decisions made when creating it are essential tasks for engineers and design professionals. As summarised by Otey et al. [1], previous researchers have determined that 48\% of CAD models fail during design exploration [2] and according to the 2013 State of 3D Collaboration and Interoperability Report, 49\% of engineers spend more than 4 hours per week repairing design data with $14 \%$ spending more than 24 hours per week [3]. The same report states that $32 \%$ of organizations miss deadlines due to design data problems [4].

Mass-customization has been a steady driving force to capture and automatically utilize such engineering knowledge. Many companies have, for instance, put significant efforts to parametrize CAD-models to quickly and accurately respond to changes in product requirements and specifications. This has caused engineers to not only focus on developing single products but product families with wide and flexible design spaces. In parallel to parametrized CAD-models, knowledge management and knowledge based engineering (KBE) systems have for decades strived to capture, digitize, and automate the application of this kind of knowledge within product and production development.

The problem has also received academic attention. Visualization of interdependencies of elements in CAD-models is an active research subject. Kozlova et. al. reviewed how graph visualization can be used for CAD-models of architectures [5]. In that work prototypes for interactive graph visualization were also developed. The focus of that work was the visualization of the graphs and functions. Tsygankov et. al. [6] studied how to semantically represent the building process of CAD assemblies containing multi-body components. Camba et. al. [7] developed methods to traverse CAD-models of components to identify and visualize dependencies between features. Marchenko et.al. [8] developed a tool to represent CAD-models as graphs in CATIA, which considered how the elements of the CAD-models were connected not only as parent/child relations but also through mathematical formulas. Graph rendering and filtering based on CAD-models, spread sheets and KBE-rules in order to support connectivistic learning in product development has been presented by Johansson et al. [9]-[11].

Even though KBE-systems have gained much attention through the last three decades, industries still find them hard to develop and even harder to maintain over time. $t$ has been stated that arbitrarily small changes in the initial conditions of chaotic systems can lead to large changes in the results [12]. A KBE system can indeed be deterministic chaotic. For instance, changing angular dimensions in a CAD-model may cause arcs to flip inside-out (chaos due to topology). Also, slightly changing initial parameter values may trigger rules 
with mathematical expressions combined with if-statements (which are common in KBE-systems) so that the output is far different than the original (deterministic mathematical chaos). Further, when considering how manufacturing equipment is affected by changes further increases the chaotic effect. Consequently, in this paper, we suggest taking a connectivistic view on knowledge to contribute on defining a better way to help industries to maintain their product knowledge continuously.

We start with an introduction to connectivism, its principles and cornerstones, were singularities detected in applying connectivism to KBE context are highlighted. Then a brief review is given on how product knowledge is formalized and represented. After that, our connectivistic view is introduced by way of a detailed description of how three of the most common knowledge carriers in manufacturing companies (CAD-models, spreadsheets and KBE-rules) are constituted and how their constituents are connected. Then three examples are described: two where product knowledge was penetrated to gain understanding of the relations within parametric CAD-models connected to KBE-systems or spreadsheets and one where a CAD-model was penetrated to evaluate its modelling quality. Finally, the results are discussed, before the paper is concluded.

\section{Connectivism and KBE}

Connectivism is a teaching strategy embracing that knowledge in the modern society is connected, distributed and changing. This teaching strategy has been developed and applied relating to massive open online courses (MOOC), where anyone on the globe can attend the course which occurs on the Internet.

The connectivistic view of knowledge was introduced by George Siemens and Stephen Downs and was vividly described by Siemens in [13]. Connectivism is defined by Downs as "the thesis that knowledge is distributed across a network of connections" [14], and address learning that is located within technology and organizations, a learning that KBE ultimately is intended to support. Siemens defines connectivism as "the integration of principles explored by chaos, network, complexity, and self-organization theories", and introduces its nine principles (described but not numbered by Siemens [13]):

1. Learning and knowledge require a diversity of opinions to present the whole... and to permit selection of the best approach.

2. Learning is a network formation process of connecting specialized nodes or information sources.

3. Knowledge rests in networks.

4. Knowledge may reside in non-human appliances, and learning is enabled/facilitated by technology.

5. Capacity to know more is more critical than what is currently known.

6. Learning and knowing are constant, on-going processes (not end states or products).

7. Ability to see connections and recognize patterns and make sense between fields, ideas, and concepts is the core skill for individuals today.

8. Currency (accurate, up-to-date knowledge) is the intent of all connectivistic learning activities.

9. Decision-making is learning. Choosing what to learn and the meaning of incoming information is seen through the lens of a shifting reality. While there is a right answer now, it may be wrong tomorrow due to alterations in the information climate affecting the decision.

\subsection{Cornerstones of connectivism}

Five components are identified within connectivism. Central in the connectivistic view on knowledge is that learning is a network formation process [13]. Networks in this context refer to online social networks which are adaptive, fluid, and readily scalable in size and scope.

Context in the connectivistic view includes elements like emotions, recent experiences, beliefs, and the surrounding environment. Each element possesses attributes, which, when considered in a certain light, inform what is possible in the discussion. The object is tied to the nature of the discussion, framework or network of thought [13]. Context in this broad definition is not typically considered in theories for knowledge management, knowledge engineering, and KBE. However, context influences how the knowledge is implemented in KBE and how it can be understood by stakeholders.

Conduits are the mediums through which knower (i.e., experts) and seeker (i.e., knowledge consumers) communicate and through which the known entity finds expression [13]. Conduits are the facilities making the knowledge relevant, current, and available. In manufacturing companies, these conduits today include e-Mail, PLM-systems, intranet, wikis, and shared file servers.

Filtering is important to connectivists. Siemens [13] briefly reviews the history of how information has been consumed and concludes that we used to go to one source of information to get a thousand points of information (for instance newspapers). Now, we go to a thousand sources of information to create our own view. He continues by saying that we have become the filter, mediator, and the weaver of the networks. Since we as humans have a limited possibility to focus our attention (we can only do one or a few things at a time, and we just have a limited time per day) and since the amount of information and knowledge is ever increasing there is a great need for fitering the content, which (in our view) may be done based on individualized filters and current context (as defined previously).

Content is of course of central importance (even if it is told that the capacity of learning is more important than what we already know). Relevance, however, is not only about the nature of the content. The process of ensuring currency of content/information is critical to managing knowledge growth and function effectively. Content has to blend with conduit and context [13] which means that 
content should be perceived to be very close. Engineers today put much time to seek for content, but rather the content should seek for the engineers.

\subsection{Main activities in connectivistic learning}

There are four main activities that take place in the connectivistic learning process. The first step is aggregation [15], [16], where the learner searches relevant sources of information and gets into the relevant information. Second step includes evaluation and reflection of the information harvested in the first step. The second step is referred to as relation [15] and remixing [16]. In the third step the information and reflections are used to create something own out of the information. This is why the third step is called create [15] or repurposing [16]. Finally, the fourth step, sharing [15] or feed forward [16], is where the gained knowledge is shared with peers or others.

All four activities are involved when developing and maintaining KBE-systems.

\subsection{Towards an approach to support KBE by way of connectivism}

In our vision, the collaborative design of a new industrial product must progress from an interdisciplinary activity (where interdisciplinarity implies a joint mixture of disciplines, according to Lawrence [17], as cited by Serna [18]), passing through multidisciplinary until shifting up to transdisciplinary (which implies the fusion between the disciplinary knowledge and the know-how of lay people). Still, the transition stages require consolidation. In particular, this paper is a starting point on how to apply the connectivistic view of knowledge [13] to KBE.

For instance, 3D Model definition is one necessary stage in collaborative product creation. In fact, CAD models are the primary view of the product (at least in the claim of the Model-Based Enterprise paradigm [19]). Thus, quality of CAD models is of capital importance for quality of products. However, sectorial approaches to control and improve quality of CAD models have proved incomplete thus far.

To apply the connectivistic view to knowledge-based engineering-while assuming that connectivism is an abstract philosophy of knowledge - this research was aimed at proving that it is possible to adapt such a mindset to further enhance KBE systems and processes to keep them alive and up-to-date. At this end, we explore the five areas described before: network, filters, context, content and conduits. Thus, this research is not intended to contribute to the theory of connectivism. On the contrary, our goal is exporting the fruitful results of this theory to contribute to develop the way engineering knowledge is treated and to keep it alive and up-to-date, i.e. viewing KBE as a continuously ongoing learning process.

Our practical challenge is developing new tools to make sure that the background knowledge of the engineering processes is available for its stakeholders throughout the product life cycle. Since the knowledge is stored in very different ways, and the stakeholders need to process the information in different ways, we need a connectivistic approach. The methods developed during the research project are far from ready and much work must still be done to make them readily available to the engineers in the global company. In this first stage we are running experiments to demonstrate both the validity and the compatibility of different approaches to check, store and retrieve pieces of engineering knowledge.

\section{Knowledge Based Engineering as a connectivistic learning process}

Engineering design problems are solved through iterations between synthesis and analysis phases. Design proposals developed through creative processes are evaluated against requirements. During new product development processes, much of the trial and error loops occur as a part of the learning of how to solve the problems connected with the product. The knowledge developed and formalized during these trials is referred to as product knowledge [20]. When the product matures, a set of tested solutions emerges. This set is reviewed when the customer demands the product for a different set of requirements. When such a set of solutions exists the synthesis phase gradually turns into a search for existing solutions that can be combined to solve new problems. Also, when the product matures the way of testing the product to requirements is formalized and can sometimes be skipped based on an inductive way of reasoning, i.e., based on experience it can be concluded that the new solution will fit. Proven and formalized knowledge can be handled as a design platform as described in [21]. In the most mature state of a mass-configured product, the processes for developing a variant based on user requirements are well defined. In such state, computer support can be utilized in synthesis and analysis phases to a great extent, which is referred to as knowledge-based engineering (KBE).

KBE is a method to synthesize design proposals and has been defined as a technology based on the use of dedicated software able to capture and systematically reuse product and process engineering knowledge, with the final goal of reducing time and costs of product development by automation of repetitive and non-creative design tasks, and support for multidisciplinary design optimization in all the phases of the design process [22]. KBE is also said to be the integration between artificial intelligence (AI) and computer-aided engineering in general, and computer-aided design (CAD) in particular, see Figure 1. Artificial intelligence is a set of methods and models from the computer science research field that support flexible modelling of concepts and techniques for logical reasoning, while computer-aided design includes tools and methods to model geometry and product structures. 


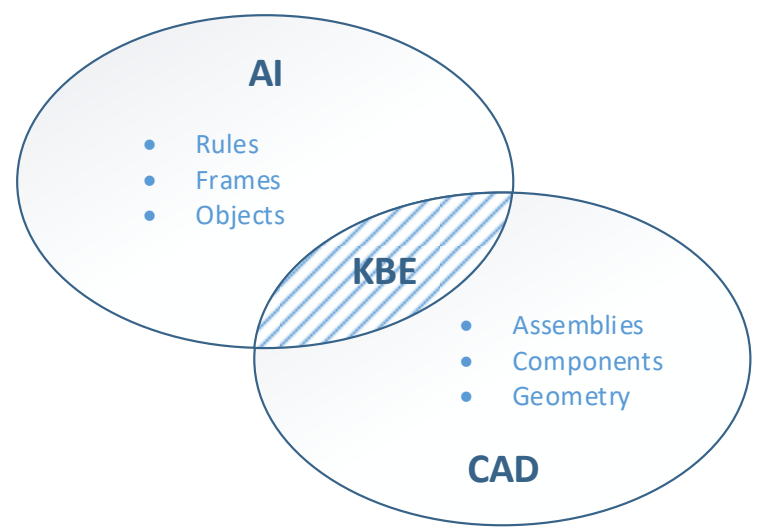

Figure 1: KBE systems from a technical perspective: computer programs containing knowledge and reasoning mechanisms augmented by geometry handling capabilities to provide engineering design solutions. Adapted from [22]

As can be concluded from these definitions, CAD-models are part of the represented engineering knowledge. They result from applying the engineering knowledge and define how the product is to be constituted and embodied. The logic and rules can be embedded into CAD-models but also stored in spreadsheets or knowledge-bases with facts and rules.

\subsection{Aggregate, relate, create, share}

Knowledge-based engineering can be seen in the context of knowledge management where KBE is a subset of knowledge engineering which is a subset of knowledge management [22], see Figure 2. When observing the KBE-overview from Rocca with the eyes of a connectivist the main activities within connectivism becomes salient, even if there are some overlaps: Problem identification and Knowledge capture and formalization together stands for Aggregation and Relation, KBE application development stands for the Creating step and Application deployment and integration stands for Sharing. It is necessary for these four activities to work fluently for a mass-customization manufacturing company to stay competitive.

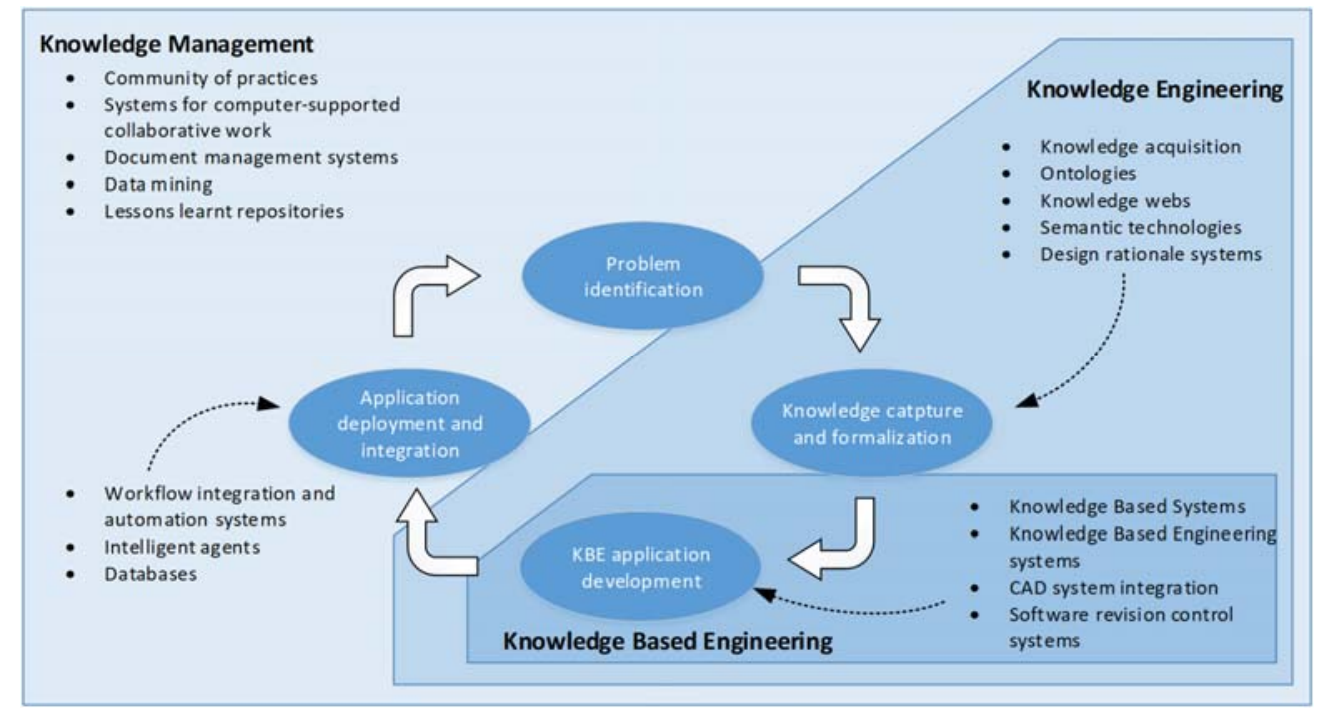

Figure 2 : Components of knowledge management including knowledge-based engineering. Adapted from [10], [22].

\subsection{Embracing chaos, complexity and self-organization}

Siemens' definition of connectivism includes chaos, complexity and self-organization. These three ingredients are prominent in product development projects. First, engineering problems are typically ill-structured [23] because they lack clear ways of testing any suggested solution, the states of the problem are hard to represent, and the knowledge of the problems is hard to capture and represent. This has led to an abundance of different information models capturing sub-aspects of the product. These information models are stored in different file formats and treated in different software applications. For example, the geometrical aspects of the product are modeled in CAD-systems while economical aspects may be modeled in spread sheet applications (we have different file formats for CAD-models and different file formats for spread sheets). The diversity of information models and digital formats form a complex body of information that in total makes for a model of the product. This complex body of information in turn may be of a chaotic nature, i.e. making small changes at one aspect of the model may cause big and unexpected effects on another aspect. The deterministic chaotic behavior originates both from the AI and from the CAD sides of the KBE system (see Figure 1). The AI side is constituted by large sets of rules that 
oftentimes are non-linear and non-continuous (e.g. mathematical expressions combined with if-statements). The CAD side is constituted by large sets of geometrical entities related in different mathematical aspects. These entities may be suppressed or reactivated in vast number of combinations. These entities may also have a nonlinear response to changes (e.g. arcs may turn out-side in when changing angular measures).

Self-organization takes place, according to the connectivistic view, due to pressure to change, which may "arise from different sectors of a system. At times it is mandated from the top of a hierarchy, other times it forms from participants at a grass-roots level" [13]. The change pressure connected to a KBE-system typically stems from changes in legislation, new technologies and changing markets which all initiates changes of CAD-models and rules in the KBE-system. The closed loop of four activities as presented in Figure 2 accounts for the change pressure.

\subsection{Networks and content}

In the knowledge management technologies, as seen in Figure 2, networking is embraced through the community of practices, systems for computer-supported collaborative work, ontologies and knowledge webs. Traditionally, and as seen in Figure 2, these networks enabling technologies are not considered to be a part of knowledge-based engineering system. Content is realized by design rationale, rules and geometrical models. It is vital for the content to be accurate and responsive to changes in the network.

\subsection{Introduction to graphs and filtering}

Graphs are central in knowledge modelling and are briefly introduced here to support the reader. A graph G(N, E) is a set of nodes (N) and edges (E). The nodes represent entities of interest, and the edges represent how they are connected as tuples of two nodes, where the first one is the source node, and the last one is the target node [24]. When two nodes are connected through an edge, they are said to be neighbors. The degree of one node is defined as the number of neighbors it has, i.e., how many edges are pointing in and out from it. In-degree accounts for how many neighbors a node depends on (parent), and out-degree accounts for how many neighbors are depending on it (child). Nodes and edges can be labeled indicating what type of entity a node represents and what kind of relation an edge represents. Further, attributes can be assigned to nodes and edges. For nodes, attributes indicate what state the represented entity has, and for edges, the attributes indicate what state the relation between two nodes has.

In the context of this paper, filtering means retrieving graphs by reducing or combing datasets, which is achieved by the application of set theory. The fundamental set of Boolean operations (union, difference, and section) can be applied to nodes and edges and combined so as to produce queries. If the underlying data is stored in a relational database, it is possible to use SQL for filtering. However, a growing number of graph databases and graph querying languages under development support extensive ways of managing and filtering large graphs applying graph theory. The latter method of storing data has proven to outperform relational databases when the data is highly connected [25]. Graph databases are applicable when the connections between the stored entities are equally or more important than the entities themselves.

\section{Dissecting the constituents of engineering knowledge in parametric design}

To develop tools and methods to support a connectivistic view of KBE it is important to know what type of content will fill the network and how this content may be connected. In this study, we focus on parametric CAD-models as such, and parametric CADmodels controlled by spreadsheet applications or by KBE-systems (or both). There are three ways in which a parametric CAD-model (further on it is assumed CAD-models to be parametric) can be controlled. First, spreadsheets can be connected to CAD-models to define family tables, i.e., sets of similar components derived from a single parametric CAD-model. That is an efficient way of handling parametric design of, for instance, fasteners, washers or other standard components. Second, KBE-systems can be connected to CADmodels to execute rules that update the CAD-models. This is useful when handling components and assemblies that are free to change based on customer requirements. A third way is to add formulas into spreadsheets and add them to the geometry rebuild process. In this latter case, the spreadsheet application turns into a KBE-system.

We will take a close look at these three types of containers of engineering knowledge (CAD-models, spreadsheets, and KBEsystems) to find their fundamental constituents and to see how they are connected. Then we apply graph theory and filters to put the basics to further studies in knowledge and complexity management.

\subsection{CAD-models}

The information model of a CAD-model can be constituted in many ways and differs between CAD-systems (therefore neutral CAD-formats are needed). Still, in Figure 3 a schematic information model shows the commonly agreed fundamental constituents. An assembly is, as seen in the figure, composed of instances of parts which can either be components or assemblies (the terminology used in the literature differs somewhat between what is a part and what is a component, here a component is a piece made from one material, a part can be either one component or a composition of components). Components are made up from at least one feature while assemblies may contain features or not. Components and assemblies may comprise parameters which are carriers of base type data such as Booleans, integers, doubles or text values packed with a name. Assemblies and components may comprise equations, which are mathematical expressions involving parameters. The most common type of feature in CAD-models is geometrical features. Geometrical features are the composition of entities which may be one-, two- or three-dimensional geometrical elements, such as 
points, lines, curves, planes, and surfaces. Geometrical dimension or constraints are a particular type of parameters that make reference to geometrical entities to control their definitions.

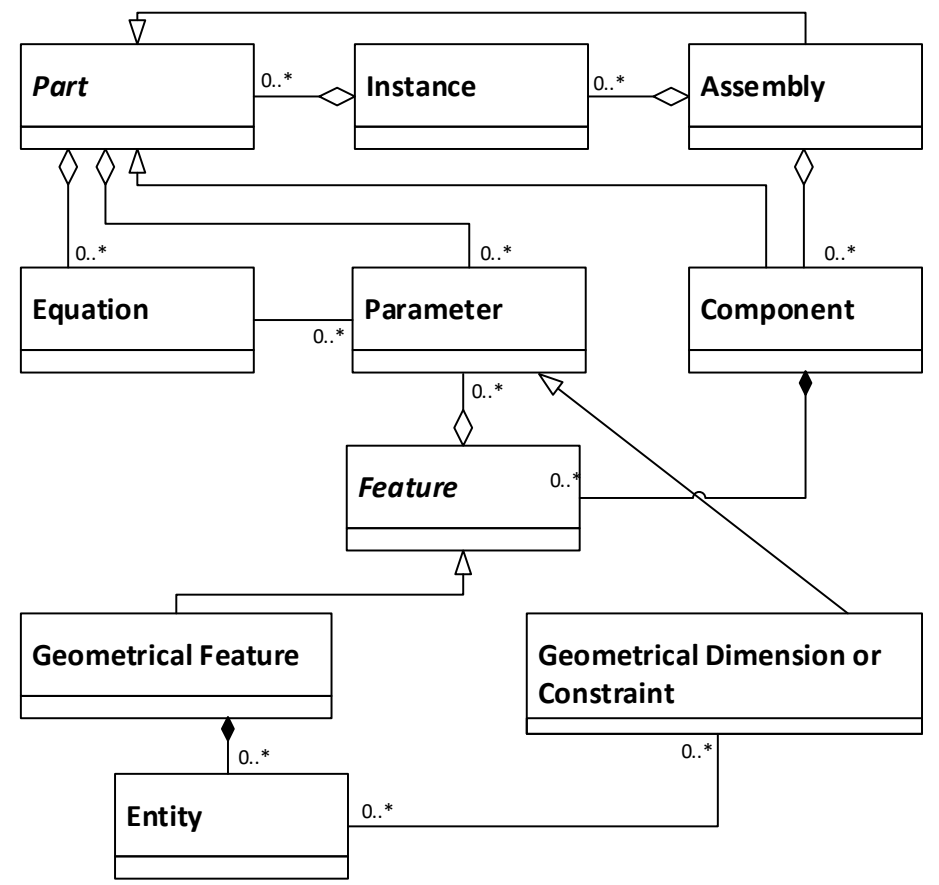

Figure 3: Information model of CAD-model.

There are several types of relations in a CAD-model. As seen from Figure 3, components are related to assemblies as "part-of" relations. Features, parameters, and equations are also "part-of" components, and entities are "part-of" features. These easy-tounderstand relationships are often visualized in the CAD-system through a "model tree". A typical model tree is shown in Figure 4, which contains a top assembly (Assembly1) having three instances of parts (2 SubAssembly1 and 1 SubAssembly2). SubAssembly1, in turn, is constituted by two instances of Component1, which is made up from Feature1 and Feature2 (Note that not all CAD-systems show the instance-level in the model tree, i.e., the nodes SubAssembly1 (1), SubAssembly1 (2), and SubAssembly2 (1)).

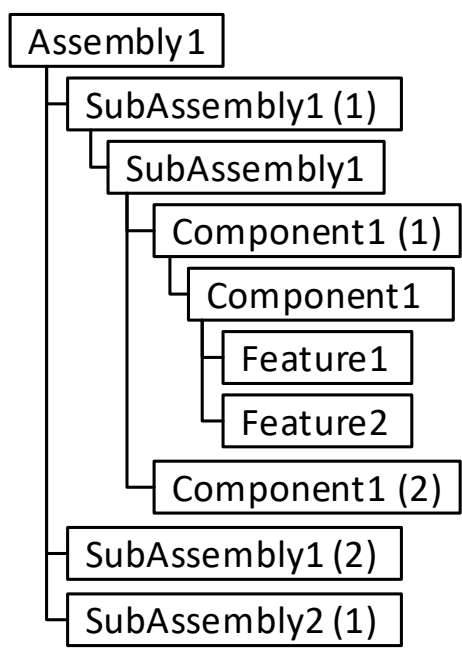

Figure 4: A typical model tree in CAD-systems only show "part-of" relations.

Other than "part-of" relations exist in CAD-models which are not that obvious but are interesting to engineers when developing and maintaining the models. An example of such type of relationship is the references between geometrical entities conveyed through geometrical dimensions or constraints. The geometrical engines efficiently manage local references but cross references between entities belonging to different features result in hierarchical dependencies named as parent/child relations (where, for instance, deleting the parent feature will cancel the child one). Another example of relationships important to engineers involves the intent of the CAD-model. 
Most CAD-systems allow adding logic to the CAD-model through equations, which may govern the parameters (like the number of instances of a repetitive pattern), the geometrical dimensions (that govern the size or even the topology of the CAD-model), or other geometrical relationships (like Boolean flags that may govern the parallelism or perpendicularity between two entities). We name them as mathematical relations. These relations could also be viewed as parent/child relations if they are unary expressions. The relationships are modeled as edges in the graphs per Table 2.

\subsection{Spreadsheets}

Spreadsheets are frequently used within engineering design to store and manage information regarding the product and are a part of the product model. Spreadsheets may, as mentioned, be connected to CAD-models as design tables or as a part of the geometrical build process as "analysis" features. The reason for adding spreadsheets as a part of the product model is the flexibility to model information. The central concept in spreadsheet applications is the cell. In Figure 5 a schematic information model of a spreadsheet is drawn. Cells reside, as seen in Figure 5, in worksheets and worksheets reside in workbooks. A cell may contain a formula that refers to other cells. Formulas act as functions with several possible input cells but with one output only, which is displayed in the cell to which it belongs.

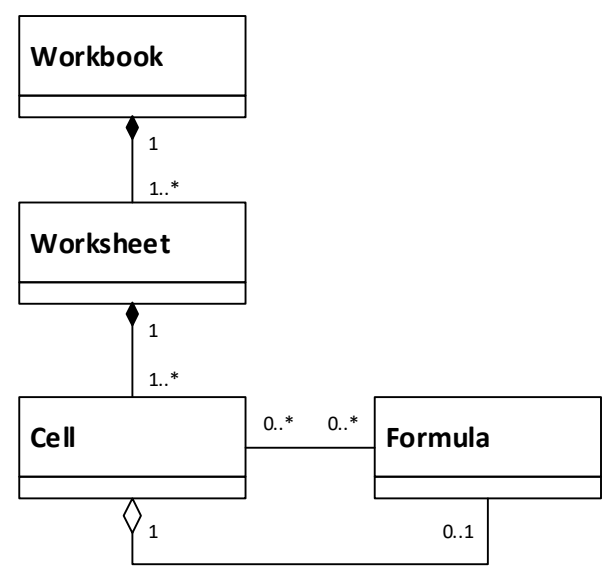

Figure 5: Information model of spreadsheets.

When connected to a CAD-model we can view cell values as facts, and formulas as rules in a KBE-system according to the definition of $\mathrm{KBE}$ in [22], where the inference engine is then realized by the spreadsheet application (which automatically updates the cell and displays the result when the linked cells are modified).

\subsection{Knowledge-bases}

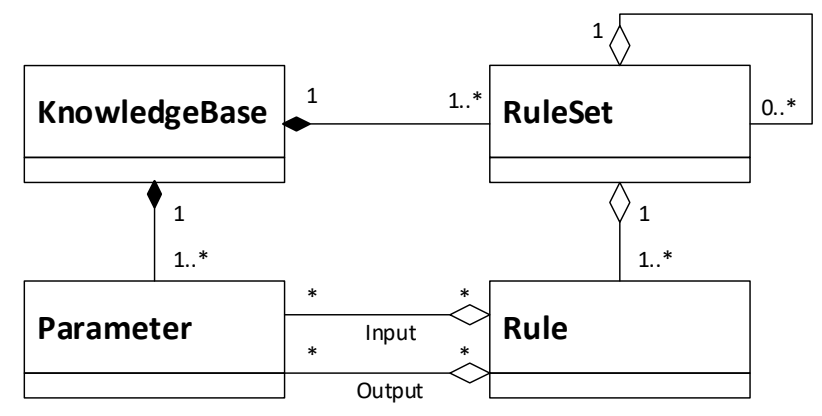

Figure 6: Information model of knowledge-bases.

KBE-systems gained attention for the CAD community during the last decade and have been integrated to several CAD- systems. Standalone KBE-systems also exist. The most interesting class in a KBE-system is the Rule class. Rules reside, as seen in Figure 6, in rulesets, while rulesets reside in KnowledgeBase. Rules consist of parameters and act as functions with one or several input parameters and one or several output parameters. Note that the parameters in Figure 3 are a subclass of parameters in Figure 6 since the parameter class in a knowledge-base tends to be more general than in a CAD-model.

\subsection{Graphs and filters}

The constituents of CAD-models, spreadsheets, and knowledge-bases; and their relations together form networks that can be represented as graphs. These graphs can be filtered, and visualized to gain insight to the model. They can also be used as a foundation 
for navigating rationale as described in [26]. To achieve all that, it is useful to make the graph meaningful by adding attributes to nodes and edges. Attributes attached to the nodes and edges in this paper are listed in Table 1.

The EdgeType attribute is what distinguishes the graphs presented in the paper from the usual model-trees in CAD-systems, as it makes it possible to model how the entities are connected. When reviewing the class diagrams in Figure 3, Figure 5, and Figure 6 five different types of couplings are found, these are listed in Table 2. The values in the first column of that table are used as valid values for the EdgeType attribute, as much as labels for the edges.

Table 1: Four attributes were added to develop the graphs in this paper.

\begin{tabular}{lll}
\hline Name & Applies to & Description \\
\hline URI & Nodes & $\begin{array}{l}\text { Unique Resource Identifier. } \\
\text { Includes file path and the internal } \\
\text { path to the represented entity. }\end{array}$ \\
$\begin{array}{l}\text { Label } \\
\text { EntityType } \\
\text { EdgeType }\end{array}$ & $\begin{array}{l}\text { Nodes, Edges } \\
\text { Edges }\end{array}$ & $\begin{array}{l}\text { Type of entity } \\
\text { Type of relation as in Table 2 }\end{array}$ \\
& & \\
\hline
\end{tabular}

Table 2: Five types of relations are identified within CAD-models (the two marked by * are the only relation types that are undirected). Connected constituents are defined in Figure 3 and Figure 5.

\begin{tabular}{lll}
\hline Relation type & Connected constituents & Realized by \\
\hline Part-of & Entity $\rightarrow$ Feature & Feature entities \\
Part-of & Feature $\rightarrow$ Component & Component features \\
Part-of & Component $\rightarrow$ Assembly & Assembly instances \\
Kind-of & Instance $\rightarrow$ Part & Instance \\
Connection & Entity $\rightarrow$ Feature & References in feature \\
Mathematical & Parameter $\rightarrow$ Parameter, & Expressions in \\
& Cell $\rightarrow$ Cell & equation, Formula \\
Connection* & Entity $\leftrightarrow$ Entity, Part $\leftrightarrow$ Part & Geometrical \\
& & constraints \\
Spatial* & Entity $\leftrightarrow$ Entity & Location \\
External & Cell $\rightarrow$ Parameter & Design Tables \\
Connection & & \\
\hline
\end{tabular}

Filtering of information in the graphs is what results in the contextualization of knowledge, although — due to the innovative nature of design - there will never be a set of universal filters; however, some filters may be more general or more frequently used than other. Here we suggest three sets of filters. The first set of filters is the combination of retrieving nodes of types Geometrical Dimension, Parameter and Cell (i.e., nodes representing entities that control the design) and that are of degree 1 . The entities represented by the retrieved node of such filters are the entry points for the CAD-model: changing any of their values impacts the design. In everyday language, we call these entities design parameters.

The second suggested set of filters involves filtering on Part-Of, and Kind-Of edges which yields the model tree as represented in the CAD-systems (it is a combination of the "Assembly-tree" and "Part-tree") which can be used as a support to evaluate the complexity of CAD-models. The output from this filter corresponds to the product variant master [27] which is a tool supporting the development of configuration systems.

The third set of filters involves filtering edges on External connections. When applied, they yield the interfaces between the CADmodel and its governing design tables. Further, if adding edges of types "Mathematical" gives the entire set of logic for the CAD-model. The nodes resulting from that filter represents the logical part of the product model.

\subsection{Rendering graphs}

To get a seamless overview of the engineering content of CAD-models and their connected spreadsheets and knowledge-bases, they can be analyzed using the theory in the previous sections to render graphs. In our implementation, there are three algorithms used to do the analysis, the first one traverses the CAD-model, and its sub-models to extract the nodes and edges. The second algorithm analyses any spreadsheet connected to the CAD-models to extract nodes and edges within them and in connection to the CAD-model. The third one loops through all rules and connected parameters to extract nodes and edges.

A simple example demonstrates the output from these algorithms. Let say we have a CAD-model containing a box with a hole through (see left of Figure 7). The model-tree contains merely two extrude features, one for the box and one for the hole. The two features are based on their corresponding parametrized sketches, which are shown in the model tree (see the center of Figure 7). The dimensions of the box are controlled by a design table, so that height, width, and length are controlled by the cells in a connected spreadsheet. Two configurations (or product instances) are defined (Default and Config1). The set of values for the first configuration 
is in turn controlled by a "base value" that is a sort of "scale" that applies to all the three parameters and is stored in a cell in another spreadsheet (see the right side of Figure 7).
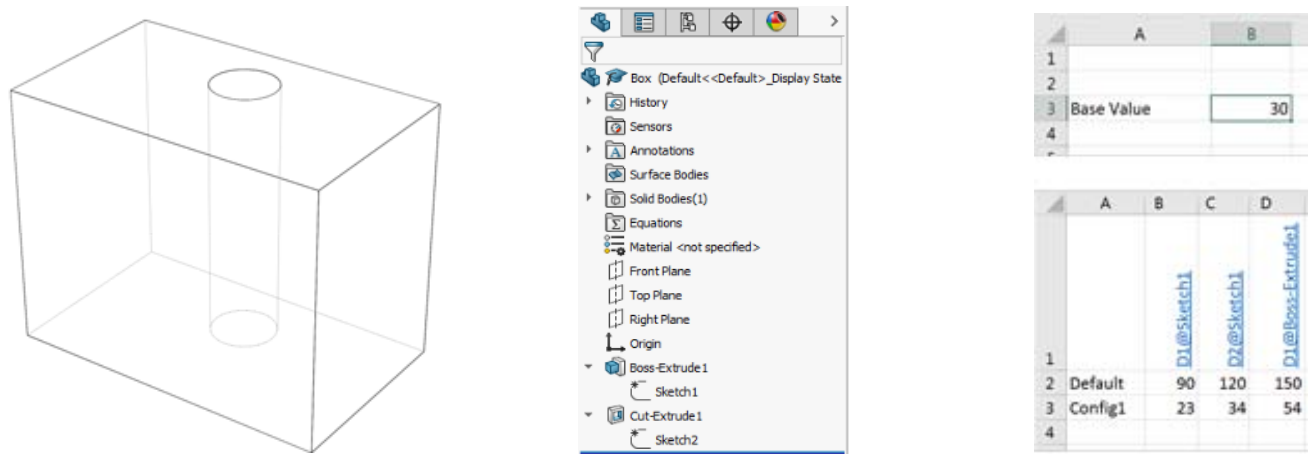

Figure 7. Simple CAD-model of a box with a hole with a corresponding model tree (left and center) and a connected spreadsheet, base value controls Default configuration (right).

Applying the algorithms to the simple box-with-hole example yields a graph with 22 nodes and 28 edges. The graph is visualized in Figure 8 with the Yifan Hu layout algorithm [28]. There are seemingly duplicate nodes (for instance two D1) this is because of using short names on the nodes (Label attribute). In fact, the nodes are unique with the URI, thus, displaying the full names would solve this apparent duplicity but make the figure hard to read.

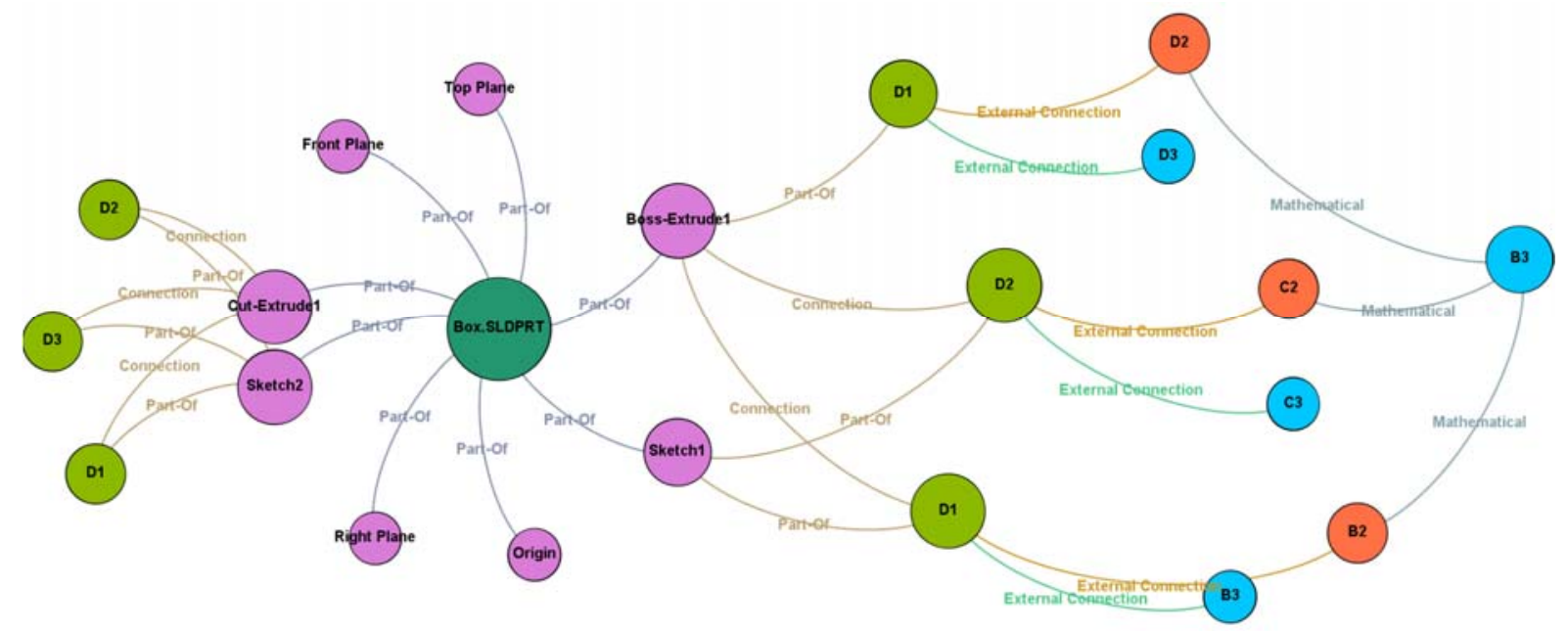

Figure 8: Graph illustrating the relations in the CAD-model, design table and in between. 22 Nodes and 28 relations.

Applying a filter to retrieve design parameters results in 7 design parameters: the nodes D1, D2 and D3 (green) to the left and B1, B2, B3 and the other B3 (blue) to the right in Figure 8. This is interesting as it indicates that every value in a design table is indeed a design parameter. Applying a filter to retrieve part-of relations yields two graphs one with the CAD-model and one with the spreadsheet. Using a filter to extract the mathematical model yields a graph with nodes B2, C2, D2 (orange) and B3 (blue), which represent the logical part of the product model.

\section{$5 \quad$ Eliciting knowledge about relations in parametric design using graphs}

To verify the concepts presented in the previous sections a prototype software was developed and applied to two real-life examples: hot runners for injection molding, and truss joints in earthquake-proof buildings. These cases illustrate how graph rendering and filtering can be applied to gain knowledge about CAD-models that are complex regarding variants and regarding numbers of rules.

\subsection{Hot runners for injection molding}

The first company where the software was tested develops and manufactures heated runner systems for injection molding of plastic components. The company has automated their engineering processes to some extent as described in [29]. One reason to analyze the company's CAD-models was that the product is suitable for design automation, in spite that every produced hot runner system is unique. The runner systems differ in layout. See for instance Figure 9, where an example of the H-shaped layout is shown. There are also Xshaped layouts, circular layouts, and custom layouts. The runners are connected to the tooling cavity through in-gates. The number of in-gates is up to 48 for a single system. There are five series of in-gates that all can have two different types of bushings (with lengths ranging up to $600 \mathrm{~mm}$ ), and have up to 9 different types of end caps. This led to the automation of the CAD-models based on 
parametrized families managed through interconnected spreadsheets used as design tables. Due to the combinatorial complexity of the product, mathematical equations were added as equations in the CAD-models as well as in the spreadsheets. This approach made the CAD-system unstable so that when adding several instances of the in-gates (the protruding parts in Figure 9 and CAD-model in Figure 10) it eventually crashed after a long number crunching time (sometimes up to 40 minutes). It did not always crash, so the engineers tended to wait and hope for it to go through. The crashing problems were eliminated when reforming the CAD-models as described in [29]. In the subsections, we describe the CAD-models, spreadsheets and the results of rendering and filtering graphs.

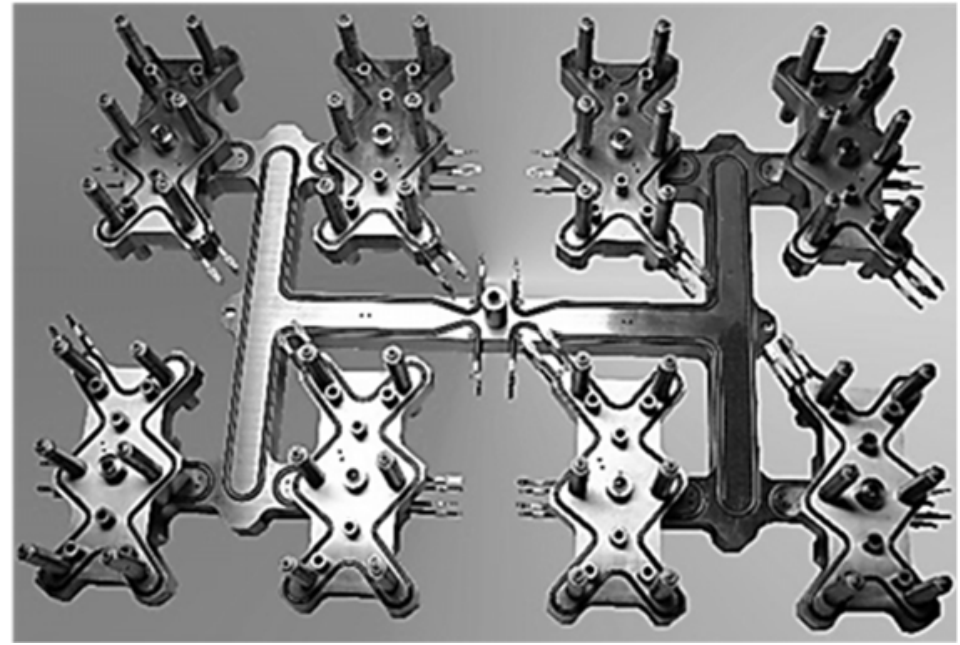

Figure 9: Hot runner system with 48 gates in X-layout [29].

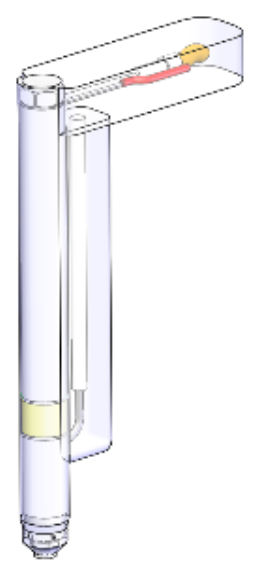

Figure 10: This seemingly small and easy CAD-model caused the CAD-system to crash repeatedly.

\subsubsection{CAD-models}

The targeted CAD-model is created in Solidworks and consists of five sub-parts of which only one is an assembly (which in turn consist of three components). In total, we are talking about seven components in two assemblies, which seems very little to make a CAD-system to collapse. The vast number of variants for each component is what makes it so difficult. Besides, the product is to operate in much higher temperature than it is produced, which resulted in several temperature configurations for each component (although this was not considered in the test reported here). The top-level assembly contained 42 equations controlling features on all levels and was connected to a spreadsheet as a design table.

\subsubsection{Spreadsheet}

The spreadsheet connected to the CAD-model as a design table was a Microsoft Excel spreadsheet and included all possible combinations of the part components. The combinations were added using formulas in the spreadsheet so that design table updates when changing certain cell values (this method works for engineer-to-order products). In total, the design table contained 1248 cells, and there were additionally 46 cells with formulas to adjust the values in the design table.

\subsubsection{Graph extraction and content filtering}

The prototype software, which was developed in the Microsoft .NET platform, took 2 minutes to generate the entire graph. The output data was stored in Neo4j graph-database. This database was queried using the Cypher querying language for graphs [30]. The resulting graphs from the queries (the three filters mentioned previously) were exported as Graph Modelling Language (.GraphML) 
which is a general, XML-based language, to store graphs in a standardized way [31]. To visualize the graphs several freely available software applications were tested. Due to the size of the graph Gephi [32] with the Yifan Hu [28] and Force Atlas 2 [33] layout routines proved to work. The graph contains 3932 nodes (47\% formula, $28 \%$ geometrical dimension, $17 \%$ feature, $2.9 \%$ instance, $2.2 \%$ cell, $0.7 \%$ Component, $0.6 \%$ Assembly, $0.2 \%$ parameter) connected in 11321 relations.

When applying the filters, it showed that there are 1165 entry points of which the majority are of Geometrical Dimension type, further filtering shows that there are 12 parameters and 86 cells. Although the academic experiment proved the usefulness of the approach, for industrial use, these entities should be managed, and the information regarding them should be made accessible to retrieve to engineers. Filtering for product structure made it possible to automatically retrieve a product-variant-master [27]. Seven subgraphs occurred when filtering for mathematical relations four subgraphs represented equations in the CAD-model and three represented formulas in the spreadsheet.

By rendering, visualizing, and filtering graphs from the CAD-model, it was possible to identify all entry points, simplifying the product structure and structuring the represented knowledge in equations in the CAD-model and its connected spreadsheet. Thus, the graph proved useful to give a clue of why the parametrized model became so difficult to handle.

\subsection{Truss joints in earthquake-proof buildings}

Another prototype software was developed and applied to an industrial company that develops and manufactures power plant solutions. The power plants partially consist of steel trusses, which must be earthquake proof. The company tested to automate the construction of truss joints to cut lead-time. The result of the tests was a set of CAD-models with such a huge number of rules embedded into them that it was perceived hard to manage their design content. In this study one CATIA CAD-model consisting of one component was targeted. This seems very little and easy to overview. Still, the vast number of rules for each component (stored in the CAD-model) makes it difficult to manage the CAD-model. The CATIA Knowledgeware KBE-rules connected to the CAD-model as formulas, checks, and design tables included all possible combinations of the component.

\subsubsection{Graph extraction and content}

In total, the knowledge-base consists of 82 formulas, seven rules, four checks and three design tables. The prototype software, developed in the Microsoft .NET platform, took 469 seconds to generate the entire graph. The output from the routine was stored in Neo4j graph-database. This database was queried using the Cypher querying language for graphs [30]. The resulting graphs from the queries (the three filters mentioned previously) were exported as Graph Modelling Language (.GraphML) which is a general, XMLbased language, to store graphs in a standardized way [31]. Gephi was used to visualize the graphs.

The graph contains 435 nodes connected in 824 relations. When applying the filters, there are 15 entry points of which 12 are of Geometrical Dimension type, 2 String parameters and 1 Integer parameter. Again, although the academic experiment proved the usefulness of the approach, for industrial use, these entities should be managed, and the information regarding them should be made easy to retrieve to engineers. However, the problem is challenging, as the logical model consists of 129 nodes and 125 mathematical connections. However, when applying the Force Atlas 2 [33] layout, it was seen that the logical model could be grouped into 16 clusters of which 5 clusters where non-sense since they only contain rules for the transition of KBE parameter to CAD dimension.

By applying the algorithms to render and filter graphs from the CAD-model and its connected knowledge-base, it was possible to identify its entry points and to restructure the knowledge-base to a more intuitive organization and to move its central parts out from the CAD-system.

\section{Ensuring content quality in CAD models}

So far in this paper, we have seen how spreadsheets and knowledge-bases connected to CAD-models can be penetrated to render graphs. These graphs are subsequently filtered to gain knowledge regarding the logical models that control the CAD-model. However, for the logical model rules and formulas to be able to control the CAD-model, the CAD-model itself needs to be sound and of good quality. This is vital to eliminate any unnecessary chaotic behavior of the total model of the product. In this section, we show by an example how the quality of CAD-models can be evaluated based on graph theory and filtering.

\subsection{CAD-model quality}

The theoretical model of quality is based on the linguistic model by Contero et al. [34], which defines three levels. The morphological quality level is related to the geometrical and topological correctness of the CAD model. The syntactic quality level is linked to the proper use of modeling conventions such as naming rules for features, datum, part, assembly, drawings, and layouts; layer structure and function and part/assembly parameters and attributes. The semantic/pragmatic quality level takes into account the CAD model capability for reuse and modification. CAD users have an abundant variety of modeling procedures for shaping their designs. However, experience shows that certain procedures provide better solutions than other. To provide a score of model quality, and approach inspired in the concept of rubrics has been applied.

On one hand, learning rubrics are scoring guides, constructed of descriptors or evaluative criteria (usually arranged in a table format) to set up the specifications to assess [35]. Summative rubrics are useless to determine the quality of CAD models, as they produce a final summative or global score (so they aim to sort subjects into those who pass and those who fail the evaluation), while formative 
rubrics are worth considering, as they provide feedback about the performance [36]. In fact, a specific approach to check the quality of CAD models by way of suitable formative rubrics was proved helpful to disclose the different dimensions of CAD models quality [37], although was also proved difficult to apply by using traditional "static" rubrics [38].

Computer science has been reported helpful to provide e-rubric forms with enhanced capabilities, including "anchors" (written descriptions, examples that illustrate the various levels of attainment, or work samples [39]) or the capability to become adaptable and provide metadata about the evaluation process. This is the case of the Annota e-rubric platform [40] that we developed to deal with the management of complex rubrics as those used to derive the score used in this study.

On the other hand, the capability of a model to be reused is linked to a proper modeling sequence. Different modeling strategies lead to different Parent/Child graph structures (This is the same as filtering on product structure "kind-of" and "part-of" relations). To obtain a complexity metric of these graphs, we "reused" in this context, the size and cyclomatic complexity (CC) parameters used in software engineering as indicators of source code complexity [41] where Size is represented by the number of nodes in the model, while $C C$ represents the number of independent paths through the graph and is calculated as:

$C C=e+i+u-n$

Where:

$e$ is the number of edges, that is, the number of paths between nodes.

$i$ is the number of inputs, that is, the number of nodes without parents.

$u$ is the number of outputs, that is, the number of nodes without children.

$n$ is the total number of nodes. It is the same as "size".

We have also used entropy $S$ to measure the complexity of graps. With this aim we have calculated the entropy parameter as described by Li et al. [42]:

Where:

$$
S=\frac{1}{2} n(n-1)\left(p \log _{2}(p)+(1-p) \log _{2}(1-p)\right)
$$

$n$ is the total number of nodes

$p$ is the probability of any pair of nodes to be connected that is estimated using the graph density $(d)$, that is defined as the ratio of the number of edges $(e)$ and the maximum number of possible edges in a graph with $n$ nodes $\frac{1}{2} n(n-1)$.

The hypothesis was that size, $C C$ and entropy correlate inversely with the CAD model quality so that these measures decrease when enhancing model quality. It is suggested to use the score obtained by the Annota e-rubrics platform to measure model quality.

\subsection{Graph extraction and quality assessment}

An analysis software tool was developed to process in batch mode a set of Solidworks CAD models, to extract their parent/child dependency graphs, to calculate the complexity parameters of these graphs and integrate the quality score of the analyzed models from an external spreadsheet. This tool was developed using Microsoft Visual Basic .NET and the Solidworks application programming interface (API). While in batch mode, it can successively process every model contained in a folder and its subfolders. The tool extracts the complexity parameters size, cyclomatic complexity $(C C)$ and entropy from every model.

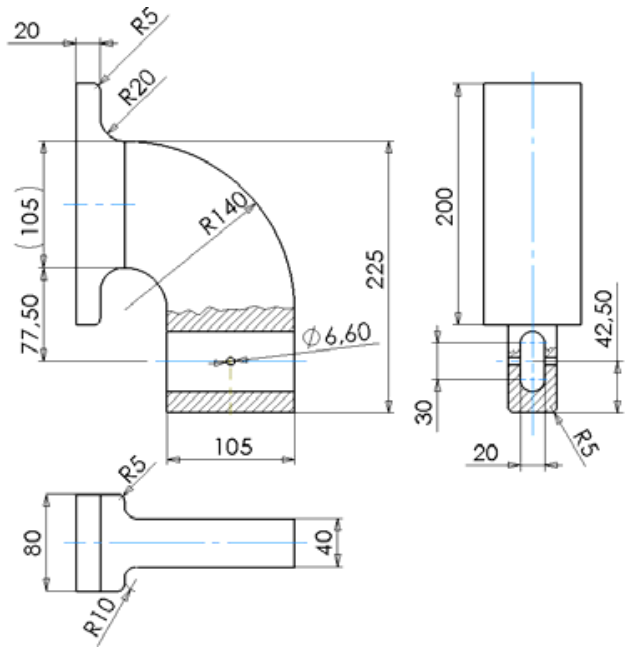

Figure 11: Part used in the experimental study. 


\subsection{Applying the method}

To test the hypothesis that both size, cyclomatic complexity $(C C)$ and entropy correlate inversely with the CAD model quality. A study has been conducted involving 47 engineering students that modeled the part defined by the drawing presented in Figure 11 . Applying the methodology explained in the previous section, the CAD models were scored on a ten-point scale (range [0, 10], where 0 means that none or an invalid model was produced, while ten means that a good quality model was obtained). Then the mean and standard deviation were tabulated for the four parameters (Table 3), and their correlations were analyzed (Table 4).

Table 3: Descriptive analysis

\begin{tabular}{lrr}
\hline Parameter & Mean & $\begin{array}{c}\text { Standard } \\
\text { deviation }\end{array}$ \\
\hline Size & 16.09 & 2.59 \\
CC & 21.74 & 5.44 \\
Entropy & 63.10 & 17.58 \\
Score & 6.86 & 1.84 \\
\hline
\end{tabular}

Table 4: Correlation results with score (alpha $\mathbf{0} \mathbf{0 . 0 1 )}$

\begin{tabular}{lll}
\hline Parameter & $\begin{array}{l}\text { Spearman } \\
\text { rho }\end{array}$ & p-value \\
\hline Size & -0.418 & 0.003 \\
CC & -0.405 & 0.005 \\
Entropy (Li) & -0.431 & 0.002 \\
\hline
\end{tabular}

As displayed in Figure 12, Figure 13, and Figure 14 all the complexity parameters show a rough negative slope when plotted against the score, as we hypothesized. Before conducting the data correlation analysis, a Shapiro-Wilk test was used to ensure that studied data set does not follow a normal distribution. Then, a non-parametric Spearman correlation test with a significance level of $0.01(\mathrm{alpha}=$ 0.01) was applied. Descriptive statistics and correlation results presented in Figure 12, Figure 13 and Figure 14 validate our hypothesis, as they show that $\mathrm{p}$-values for both parameters are less than 0.01 , and negative.

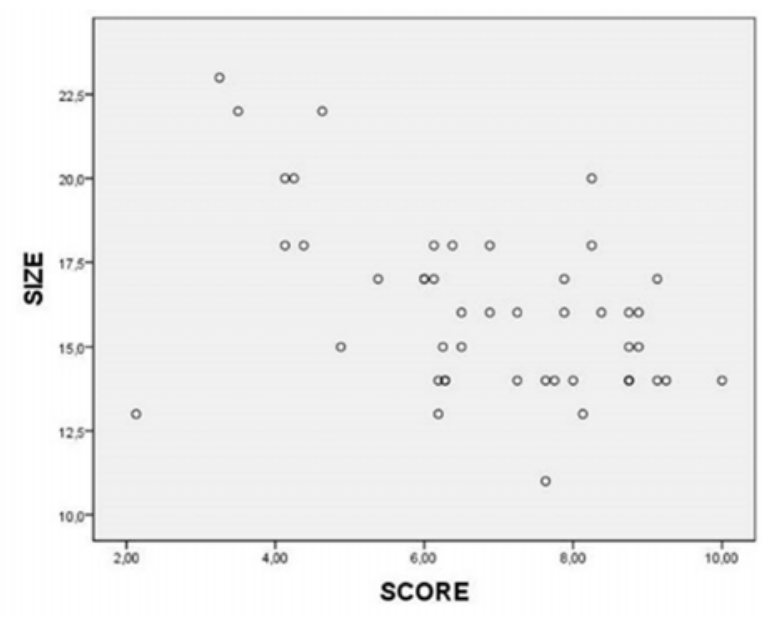

Figure 12: Size vs. quality score chart 


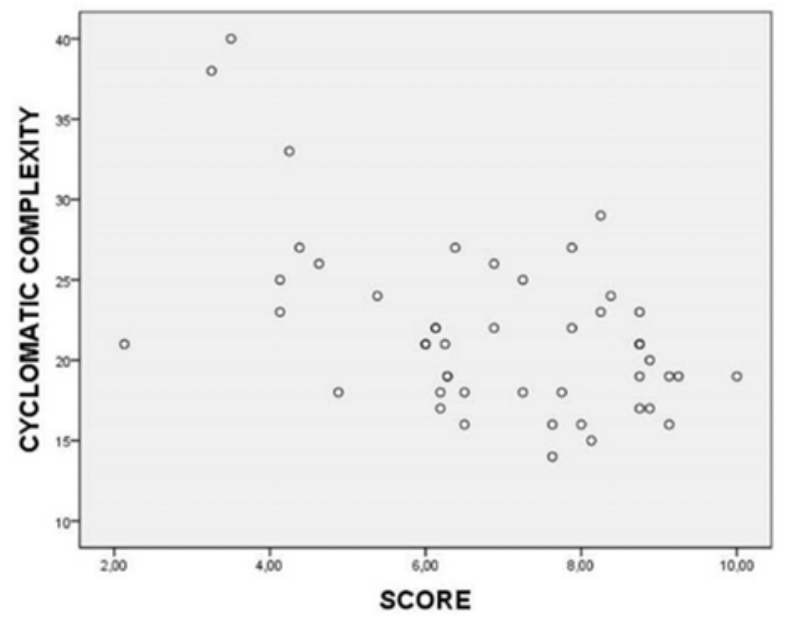

Figure 13: CC vs. quality score chart

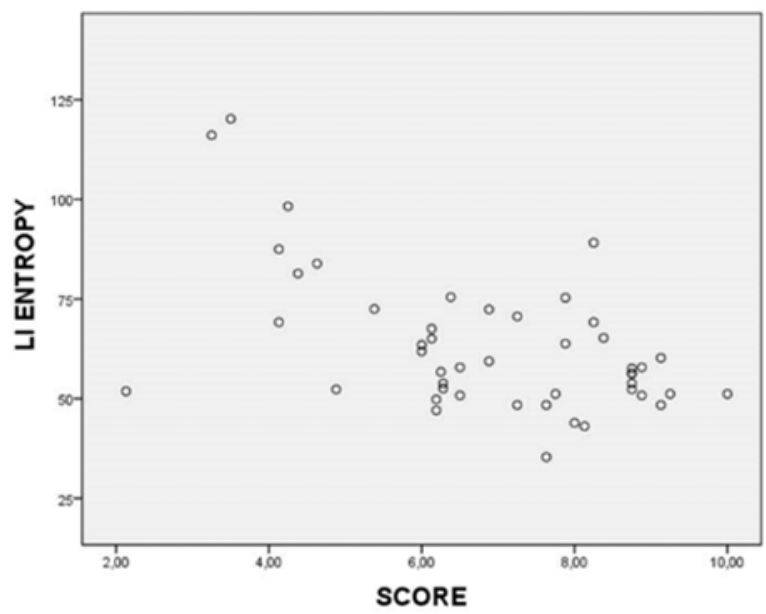

Figure 14: Entropy vs. quality score chart

The result gives insight into how to analyze the performance of CAD-models so that they can be reused and controlled by spreadsheets or knowledge-bases. High quality CAD-models (a big share of the content in the knowledge network) ensure accurate and up-to-date knowledge and reduce the chaotic behavior of the total product model.

\section{Discussion}

We discuss the results from four perspectives: the nine principles of connectivism, quality of content, context and finally the case examples.

\subsection{Applying the principles of connectivism}

When reviewing the nine principles of connectivism, it can be concluded that they can be implemented - in the context of KBE and at an industrial level — as follows:

1. Learning and knowledge require a diversity of opinions

to present the whole... and to permit selection of best approach.

Any stakeholder of the product model adding geometry, facts, or rules to CAD-models, spreadsheets, or knowledge-bases contributes to the knowledge flow. However, since the knowledge is represented in CAD-models, spread-sheets, and knowledge-bases, it may be hard to understand how these constituents are connected and why. We have seen that graphs enhance the navigation within the knowledge network. Viewing CAD-models as networks of mathematical definitions increases the granularity of the knowledge network so that connections can be made with high precision.

2. Learning is a network formation process of connecting specialized nodes or information sources.

PDM-systems serve as infrastructure to access CAD-models, spreadsheets, and knowledge-bases. However, a PDM-system only manages files and meta-data regarding the files. Adding routines to extract graphs on check-in events and storing graphs 
next to the files in the PDM system would serve as an infrastructure, to connect nodes of information sources. Each content will have its own graph stored separately next to the content file which makes the solution fully scalable.

3. Knowledge rests in networks.

The digitized knowledge stored in the KBE-system, CAD-models, and spreadsheet inherits its network from processes in knowledge management and knowledge engineering. The visualization of the network makes this more apparent. However, the real benefits of graphs lay in the way they can be navigated, and in that they can be treated mathematically to draw conclusions regarding the content.

4. Knowledge may reside in non-human appliances, and learning is enabled/facilitated by technology.

The KBE-system, CAD-models, and spreadsheets are suitable containers that facilitate storage of digitized engineering knowledge. However, quality of the content is also vital.

5. Capacity to know more is more critical than what is currently known.

As seen from the second case example it was possible to reduce the complexity of the logical model just by viewing the filtered graph. One factor that made it easy was the lay-outing algorithm that was applied in a real-time manner so that the nodes and edges were animated when applying the layout. This way of creating contextual overviews of the product models supports the organizational learning. Since knowing more is the act of connecting more, it is vital to have content that is of good quality so that the connections can be made. Also, it is important to have the possibility make the connections with high precision.

6. Learning and knowing are constant, on-going processes (not end states or products).

Viewing knowledge development as a continuously ongoing process can be enabled through versioning control of the knowledge. This is a future improvement, not considered in this paper. It would change the picture in Figure 2 so that there would be no barriers between knowledge management, knowledge engineering, and knowledge-based systems; they would be continuous sub-processes of the organizational learning.

7. Ability to see connections and recognize patterns and make sense between fields, ideas, and concepts is the core skill for individuals today.

The visualization of network and context through graphs makes it possible to see the connections and patterns of the knowledge. By filtering the graphs, it is possible to see patterns that have been hard to grasp before.

8. Currency (accurate, up-to-date knowledge) is the intent of all connectivistic learning activities.

Currency has not been targeted in this paper. However, by introducing graphs in PDM-system - as mentioned in bullet twowould make it possible to have accurate and up-to-date graphs for every CAD-model, KBE-rule, and spreadsheet readily available at every moment.

9. Decision-making is learning. Choosing what to learn and the meaning of incoming information is seen through the lens of a shifting reality. While there is a right answer now, it may be wrong tomorrow due to alterations in the information climate affecting the decision.

Viewing decision-making as a learning process is in line with viewing product development as a learning process, in which engineers indeed find themselves in a very shifting reality. Through the visualization of the network and its context, and the interactive navigation of knowledge - with easy to add knowledge content forms - an agile KBE development platform is at place supporting the flow of information.

\subsection{No one reuses chaos: keep quality up in models}

There is an important prerequisite before applying the principles of connectivism. The 3D Model definition is one necessary stage in collaborative product creation. In fact, CAD models are the primary view of the product (at least in the claim of the Model-Based Enterprise paradigm [19]). Thus, quality of CAD models is of capital importance for the quality of products. However, sectoral approaches to control and improve quality of CAD models have proved incomplete thus far. In the third case example, some parameters of the graphs were extracted to gain knowledge regarding how to develop CAD-models that can be reused and efficiently controlled by KBE-systems and spreadsheets.

The low amount of knowledge re-use in the industry is thought to be caused by the low level of standardization in the formalization of knowledge created by the design engineers. Report files content varies a lot from engineer to engineer, and it is not certain that the enterprise can safely re-use them in new projects. A more standardized way to formalize this knowledge could result in a higher re-use of the knowledge which could both save time and ensure the quality of the produced products [43]. This is supported by the rendering and filtering of graphs from CAD-models, spreadsheets and knowledge-bases.

\subsection{Achieving context}

The broad and diffuse definition of context within the connectivism may be surpassed through the development of ontologies or domain-specific languages suited to the needs of engineers. At this end, stakeholders must agree on a basic terminology useful for engineers to develop and share their concepts, models, and context. Thus, allowing for other engineers to change these models, to adapt to their context or needs. It can further be said that the KBE-system treats the elements of knowledge stored in knowledge-bases based on context as well. Sometimes a user-defined feature (composition of CAD-features) is treated as a logical element, another time it is viewed as a geometrical element being part of a drawing, and yet another time it is used in a CNC or CMM process. The term polymorphism in computer programming reflects this very technical view of context, even if the connectivistic term is much broader. 


\subsection{Reviewing the case examples}

The first case example illustrates how graph representations may help to find where to reduce the complexity of parametrized models, thus preventing catastrophic failures and/or unacceptable calculation delays. The second case example illustrates the complexity of this problem, since even an apparently simple part may convey a massive amount of design information, which will result in a very complex graph that would require powerful yet easy to use filtering capabilities. The third case example illustrates the eliciting knowledge about the CAD model quality from the complexity analysis of the parent/child graph in CAD models. This type of graph is representative of one subset of the five types of relations identified by our method within CAD-models and was used to devise engineers how to develop CAD-models that can be efficiently reused and controlled by spreadsheets and KBE-systems. It can be concluded that taking a connectivistic view on the engineering knowledge represented by CAD-models, spreadsheets and knowledge-bases made it possible to:

1. Increase the understanding of the product model by identification of entry points (design parameters).

2. Making it possible to examine the product structure and extract a product variant master.

3. Gain better understanding and possibility to retrace the logical model entangled into knowledge-base, CAD-model, and spreadsheet.

4. Devise engineers how to develop CAD-models so that they can be reused and efficiently controlled by KBE-systems and spreadsheets.

\section{Conclusions}

This paper is a starting point of applying the connectivistic view of knowledge to engineering knowledge as it is represented in product models. It was shown that by scanning the elements within knowledge-bases in KBE-systems, CAD-models, and spreadsheets, it is possible to render and filter graphs to support the connectivistic knowledge philosophy. Five areas were covered: network, filters, context, content, and conduits. Even if connectivism emerged as a teaching strategy, this research proves that it is beneficial to adopt such a mindset to enhance further the way engineering knowledge is treated and to keep it alive and up-to-date, i.e. viewing KBE as a continuously ongoing learning process. The proposed methods are far from ready and much work must be done to make them readily available to engineers in industry. However, it is already possible to apply the algorithms and methods presented in this paper to increase the understanding of product models by identification of entry points (design parameters), making it possible to examine the product structure and extract a product variant master, gain better understanding and possibility to retrace the logical model entangled into knowledge-base, CAD-model and spreadsheet, and to devise engineers how to develop CAD-models so that they can be reused and efficiently controlled by KBE-systems and spreadsheets. This helps manufacturing companies to manage and reuse engineering knowledge as valuable assets. Which according to Kennedy [20] is critical to stay competitive in the long term.

Future work includes exploration of more information models and filters, development of infrastructure to handle big and distributed graphs that together form a detailed connectivity graph of the product model, methods to utilize the graphs and client software for engineers to navigate the product model.

\section{Acknowledgments}

The work presented has evolved during the IMPACT project, funded by the Swedish Knowledge Foundation, and has been partly presented on three conferences [9]-[11]. The three conference papers show the rendering of graphs for CAD-models, spread sheets and KBE-rules together with the first case example in this article. The work has also been partially supported by grant DPI2017-84526-R (MINECO/AEI/FEDER, UE), project CAL-MBE.

\section{References}

[1] J. Otey, P. Company, M. Contero, and J. D. Camba, "Revisiting the design intent concept in the context of mechanical CAD education," Comput. Aided. Des. Appl., vol. 15, no. 1, pp. 47-60, 2018.

[2] C. Jackson and M. Buxton, "The design reuse benchmark report, Seizing the opportunity to shorten product development," Boston, MA, 2007.

[3] C. Jackson and D. Prawel, "The 2013 state of 3D collaboration and interoperability report," 2013.

[4] C. Jackson and D. Prawel, "The 2013 state of 3D collaboration and interoperability report," 2013.

[5] K. Kozlova, R. M. Sheikholeslami, L. Bartram, and R. F. Wodbury, "Graph visualisation in computer-aided design: An exploration of alternative representations for Generative-Components ${ }^{\mathrm{TM}}$ Symbolic View," in Circuit Bending, Breaking and Mending - Proceedings of the 16th International Conference on Computer-Aided Architectural Design Research in Asia, CAADRIA 2011, 2011, pp. 133-142.

[6] D. Tsygankov, A. Pokhilko, A. Sidorichev, and S. Ryabov, "The design process data representation based on semantic features generalization," in Advances in Transdisciplinary Engineering, 2016, vol. 4, pp. 127-132.

[7] C. González-Lluch, P. Company, M. Contero, J. D. Camba, and R. Plumed, "A survey on 3D CAD model quality assurance and testing tools," CAD Comput. Aided Des., vol. 83, 2017.

[8] M. Marchenko, B.-A. Behrens, G. Wrobel, R. Scheffler, and M. Pleßow, “A New Method of Visualization and Documentation 
[9] J. Johansson, "Exploring Design Content In Cad-Models And Knowledge Bases Using Graph Theory And Filtering," in Methods \& Tools for CAE - concepts and applications, 2017.

[10] J. Johansson and F. Elgh, "Applying Connectivism to Engineering Knowledge to Support the Automated Business," in 24th ISPE International Conference on Transdisciplinary Engineering, Singapore, 10 July to 14 July, 2017., 2017, vol. 5, pp. 621628.

[11] J. Johansson, “Analysing Engineering Knowledge in CAD-models and Spread Sheets using Graph Theory and Filtering," in 24th ISPE International Conference on Transdisciplinary Engineering (TE2017) : Transdisciplinary Engineering: A Paradigm Shift, 2017, vol. 5, no. 5, pp. 629-638.

[12] M. Stefik, Introduction to Knowledge Systems. Morgan Kaufmann, 1995.

[13] G. Siemens, Knowing knowledge. Winnipeg, MB]: [G. Siemens], 2006.

[14] S. Downes, "Connectivism and connective knowledge," Essays Mean. Learn. networks. Natl. Res. Counc. Canada, 2012.

[15] R. Kop, "The challenges to connectivist learning on open online networks: Learning experiences during a massive open online course," Int. Rev. Res. Open Distance Learn., vol. 12, no. 3, pp. 19-38, 2011.

[16] R. Kop, H. Fournier, and J. S. F. Mak, "A pedagogy of abundance or a pedagogy to support human beings? Participant support on massive open online courses," Int. Rev. Res. Open Distance Learn., vol. 12, no. 7 SPECIAL ISSUE, pp. 74-93, 2011.

[17] R. J. Lawrence, "Housing and health: From interdisciplinary principles to transdisciplinary research and practice," Futures, vol. 36, no. 4, pp. 487-502, 2004

[18] E. M. Serna, "Maturity model of transdisciplinary knowledge management," Int. J. Inf. Manage., vol. 35, no. 6, pp. 647-654, 2015 .

[19] J. Camba, M. Contero, and P. Company, "CAD reusability and the role of modeling information in the MBE context." NIST, Gaithersburg, Maryland, USA, 2017.

[20] M. Kennedy, K. Harmon, and E. Minnock, Ready, Set, Dominate: Implement Toyota's Set-Based Learning for Developing Products and Nobody Can Catch You. Oaklea Press, 2008.

[21] S. André, F. Elgh, J. Johansson, and R. Stolt, "The design platform - a coherent platform description of heterogeneous design assets for suppliers of highly customised systems," J. Eng. Des., 2017.

[22] G. La Rocca, "Knowledge based engineering: Between AI and CAD. Review of a language based technology to support engineering design," Adv. Eng. Informatics, vol. 26, no. 2, pp. 159-179, Apr. 2012.

[23] H. A. Simon, "The structure of ill structured problems," Artif. Intell., vol. 4, no. 3-4, pp. 181-201, 1973.

[24] R. Diestel, Graph theory, 2. ed. . New York: Springer, 2000.

[25] J. Guia, V. G. Soares, and J. Bernardino, "Graph databases: Neo4j Analysis," in ICEIS 2017 - Proceedings of the 19th International Conference on Enterprise Information Systems, 2017, vol. 1.

[26] M. Poorkiany, J. Johansson, and F. Elgh, "Capturing, structuring and accessing design rationale in integrated product design and manufacturing processes," Adv. Eng. Informatics, vol. 30, no. 3, 2016.

[27] L. Hvam, N. H. Mortensen, and J. Riis, Product customization. Springer Science \& Business Media, 2008.

[28] Y. Hu, "Efficient, High-Quality Force-Directed Graph Drawing," Math. journal., vol. 10, no. 1, p. 37, 2005.

[29] J. Johansson, "Howtomation(C) suite: A novel tool for flexible design automation," in Advances in Transdisciplinary Engineering, 2015, vol. 2, pp. 327-336.

[30] F. Holzschuher and R. Peinl, "Performance of graph query languages: Comparison of cypher, gremlin and native access in Neo4j," in ACM International Conference Proceeding Series, 2013.

[31] GraphML Team, "The GraphML File Format," 2016. [Online]. Available: http:/graphml.graphdrawing.org/. [Accessed: 07Feb-2018].

[32] Gephi.org, "The Open Graph Viz Platform.” [Online]. Available: https://gephi.org/. [Accessed: 16-Feb-2017].

[33] M. Jacomy, T. Venturini, S. Heymann, and M. Bastian, "ForceAtlas2, a Continuous Graph Layout Algorithm for Handy Network Visualization Designed for the Gephi Software," PLoS One, vol. 9, no. 6, p. e98679, Jun. 2014.

[34] M. Contero, P. Company, C. Vila, and N. Aleixos, "Product data quality and collaborative engineering," IEEE Comput. Graph. Appl., vol. 22, no. 3, 2002.

[35] Y. M. Reddy and H. Andrade, "A review of rubric use in higher education," Assess. Eval. High. Educ., vol. 35, no. 4, 2010.

[36] E. Panadero and A. Jonsson, "The use of scoring rubrics for formative assessment purposes revisited: A review," Educ. Res. Rev., vol. 9, 2013.

[37] P. Company, M. Contero, J. Otey, and R. Plumed, "Approach for developing coordinated rubrics to convey quality criteria in MCAD training," CAD Comput. Aided Des., vol. 63, 2015.

[38] P. Company, J. Otey, M. Contero, M.-J. Agost, and A. Alminana, "Implementation of adaptable rubrics for CAD model quality formative assessment," Int. J. Eng. Educ., vol. 32, no. 2, 2016.

[39] A. Jonsson and G. Svingby, "The use of scoring rubrics: Reliability, validity and educational consequences," Educ. Res. Rev., vol. 2, no. 2, 2007.

[40] P. Company, M. Contero, J. Otey, J. D. Camba, M.-J. Agost, and D. Pérez-López, "Web-Based system for adaptable rubrics case study on CAD assessmend," Educ. Technol. Soc., vol. 20, no. 3, 2017.

[41] K.-H. Möller and D. J. Paulish, "Software metrics. A practitioner's guide to improved product development," in Chapman \& 
Hall Computing Series, London: Chapman and Hall, | c1993, 1993.

[42] J. Li, B.-H. Wang, W.-X. Wang, and T. Zhou, "Network entropy based on topology configuration and its computation to random networks," Chinese Phys. Lett., vol. 25, no. 11, pp. 4177-4180, 2008.

[43] T. Hjertberg, R. Stolt, M. Poorkiany, J. Johansson, and F. Elgh, "Implementation and management of design systems for highly customized products - state of practice and future research," in Transdisciplinary lifecycle analysis of systems : Proceedings of the 22nd ISPE Inc. International Conference on Concurrent Engineering, 2015, no. 2, pp. 165-174. 